\title{
Identification Conditions for the Solvability of NP-complete Problems for the Class of Pre-fractal Graphs
}

\author{
A. V. Tymoshenko' ${ }^{1}$, R. A. Kochkarov², A. A. Kochkarov²
}

DOI: $10.18255 / 1818-1015-2021-2-126-135$

${ }^{1}$ National Research University of Electronic Technology (MIET), 1 Shokin Square, Zelenograd 124498, Russia.

${ }^{2}$ Financial University under the Government of the Russian Federation, 49 Leningradsky Prospekt, Moscow 125993, Russia.

MSC2020: 37, 90

Research article

Full text in Russian
Received March 9, 2021

After revision April 27, 2021

Accepted May 12, 2021

Modern network systems (unmanned aerial vehicles groups, social networks, network production chains, transport and logistics networks, communication networks, cryptocurrency networks) are distinguished by their multi-element nature and the dynamics of connections between its elements. A number of discrete problems on the construction of optimal substructures of network systems described in the form of various classes of graphs are NP-complete problems. In this case, the variability and dynamism of the structures of network systems leads to an "additional" complication of the search for solutions to discrete optimization problems. At the same time, for some subclasses of dynamical graphs, which are used to model the structures of network systems, conditions for the solvability of a number of NP-complete problems can be distinguished. This subclass of dynamic graphs includes pre-fractal graphs.

The article investigates NP-complete problems on pre-fractal graphs: a Hamiltonian cycle, a skeleton with the maximum number of pendant vertices, a monochromatic triangle, a clique, an independent set. The conditions under which for some problems it is possible to obtain an answer about the existence and to construct polynomial (when fixing the number of seed vertices) algorithms for finding solutions are identified.

Keywords: NP-complete problems; pre-fractal graphs; discrete problems; solvability conditions

\section{INFORMATION ABOUT THE AUTHORS}

Aleksandr Vasil'evich Tymoshenko correspondence author

Rasul Ahmatovich Kochkarov

Azret Ahmatovich Kochkarov
orcid.org/0000-0002-9791-142X. E-mail: u567ku78@gmail.com Head of the Laboratory of Maintenance Systems and Diagnostics of Complex Radio Information Systems, Professor, Doctor of Science.

orcid.org/0000-0003-3186-3901. E-mail: rasul_kochkarov@mail.ru Associate Professor, PhD in Economics.

orcid.org/0000-0002-3232-5331. E-mail: akochkar@gmail.com

Associate Professor, PhD in Physics and Mathematics.

Funding: The study was supported by a grant from the Russian Science Foundation No. 21-19-00481.

For citation: A. V. Tymoshenko, R. A. Kochkarov, and A. A. Kochkarov, "Identification Conditions for the Solvability of NP-complete Problems for the Class of Pre-fractal Graphs”, Modeling and analysis of information systems, vol. 28, no. 2, pp. 126-135, 2021. 


\title{
Выделение условий разрешимости NP-полных задач для класса предфрактальных графов
}

\author{
А. В. Тимошенко ${ }^{1}$, Р. А. Кочкаров ${ }^{2}$, А. А. Кочкаров ${ }^{2}$ \\ DOI: 10.18255/1818-1015-2021-2-126-135
}

${ }^{1}$ Национальный исследовательский университет “Московский институт электронной техники”, Площадь Шокина, д. 1 , г. Зеленоград, 124498 Россия.

${ }^{2}$ Финансовый университет при Правительстве РФ, Ленинградский просп., д. 49, г. Москва, 125993 (ГСП-3) Россия.

УДК 519.17

Научная статья

Полный текст на русском языке
Получена 9 марта 2021 г. После доработки 27 апреля 2021 г. Принята к публикации 12 мая 2021 г.

Современные сетевые системы (группы БПЛА, социальные сети, сетевые производственные цепочки, транспортнологистические сети, сети связи, криптовалютные сети) отличаются многоэлементностью и динамикой связей между ее элементами. Ряд дискретных задач по построению оптимальных подструктур сетевых систем, описываемых в виде различных классов графов относятся к NP-полным задачам. При этом изменчивость и динамичность структур сетевых систем приводит к «дополнительному» усложнению поиска решения задач дискретной оптимизации. Вместе с тем для некоторых подклассов динамических графов, которыми моделируются структуры сетевых систем, можно выделить условия разрешимости ряда NP-полных задач. К такому подклассу динамических графов относятся предфрактальные графы.

В статье исследованы NP-полные задачи на предфрактальных графах: гамильтонов цикл, остов с максимальным числом висячих вершин, монохроматический треугольник, клика, независимое множество. Выделены условия, при которых для некоторых задач возможно получить ответ о существовании и построить полиномиальные (при фиксировании числа вершин затравки) алгоритмы поиска решений.

Ключевые слова: NP-полные задачи; предфрактальные графы; дискретные задачи; условия разрешимости

\section{ИНФОРМАЦИЯ ОБ АВТОРАХ}

Александр Васильевич Тимошенко автор для корреспонденции

Расул Ахматович Кочкаров

Азрет Ахматович Кочкаров
orcid.org/0000-0002-9791-142X. E-mail: u567ku78@gmail.com начальник лаборатории систем технического обслуживания и диагностики сложных радиоинформационных комплексов, доктор технических наук, професcop.

orcid.org/0000-0003-3186-3901. E-mail: rasul_kochkarov@mail.ru доцент департамента анализа данных и машинного обучения, кандидат экономических наук, доцент.

orcid.org/0000-0002-3232-5331. E-mail: akochkar@gmail.com доцент департамента анализа данных и машинного обучения, кандидат физ.мат. наук.

Финансирование: Исследование выполнено за счет гранта Российского научного фонда № 21-19-00481.

Для цитирования: A. V. Tymoshenko, R. A. Kochkarov, and A. A. Kochkarov, "Identification Conditions for the Solvability of NPcomplete Problems for the Class of Pre-fractal Graphs", Modeling and analysis of information systems, vol. 28, no. 2, pp. 126-135, 2021. 


\section{Введение}

Количество прикладных задач в больших сетях только растет и с развитием направления анализа больших данных, увеличением числа абонентов и усложнением сетей, исследование задач на динамических графах большой размерности становится все более актуальным [1-4].

В работе [2] предложено описание динамических графов, проведен обзор известных методов их визуализации, предложены модифицированные алгоритмы с улучшенными характеристиками. Указанная последовательность соответствует термину траектории предфрактального (динамического) графа. С одной стороны, предлагаемые алгоритмы визуализации динамических графов применимы к визуализации предфрактальных графов как подклассу. С другой стороны, идентификация или распознавание социальных сетей в качестве предфрактальных графов позволит решать труднорешаемые задачи с улучшенной временной трудоемкостью, а также появляются возможности разработки параллельных алгоритмов или распараллеливания предложенных модернизированных алгоритмов.

В статье [3] предлагается метод генерации графа социальной сети, структура которого схожа с известными социальными сетями. Также авторами предложен алгоритм, учитывающий основные свойства социальной сети. Особенностью такого алгоритма стала зависимость от количества сообществ (или кластеров), а не не от количества пользователей. В терминологии предфрактальных графов в качестве сообществ выступают затравки (или блоки) разных рангов, при этом процедура порождения предфрактального графа позволяет переносить характеристики затравок на весь граф или по-другому строить граф с заранее заданными характеристиками.

Авторы [4] предложили эффективный алгоритм обнаружения и устранения коллизий в правилах межсетевого экрана в локальной сети. Древовидная структура рассмотрена в качестве структуры хранения правил политики безопасности. В соответствии с $[5,6]$ всякое дерево можно распознать в виде предфрактального графа. Адаптированный алгоритм [4] может быть применен в задачах выбора или конкурентной борьбы на предфрактальных и динамических графах.

\section{Определения и обозначения}

В настоящей работе используется общепринятое обозначение и определение графа $G=(V, E)[7,8]$. Определение предфрактальных графов соответствует работам [5, 6]. Затравкой называется связный $n$-вершинный граф $H=(W, Q)$ с непомеченными вершинами $v \in W$. Предфрактальный граф обозначается в виде $G_{L}=\left(V_{L}, E_{L}\right)$, где $V_{L}-$ это множество вершин, а $E_{L}-$ множество ребер. В процессе построения предфрактального графа формируется траектория $G_{1}, G_{2}, \ldots, G_{l}, \ldots, G_{L}$. Построенный на шаге $l$ граф называется предфрактальным графом $G_{l}$ ранга $l$.

Новыми ребрами графа $G_{L}$ называются ребра ранга $L$, а остальные ребра старыми ребрами ранга $l$, где ранг $l=1,2, \ldots, L$ - это номер в траектории $G_{1}, G_{2}, \ldots, G_{l}, \ldots, G_{L}$. Далее для предфрактальных $(n, q, L)$-графов $G_{L}=\left(V_{L}, E_{L}\right)$ используется упрощенное обозначение $G_{L}$, где $q=|Q|, n=|W|$.

На рис. 1 представлена процедура замещения вершины затравкой (3В3). Вершины графа $G_{1}$ замещаются полной 3-вершинной затравкой $H$ с произвольной смежностью старых ребер: (а) малыми пунктирными окружностями обведены вершины, замещаемые затравкой; (б) средними пунктирными окружностями обведены затравки, замещающие вершины; (в) старые ребра графа $G_{2}$ выделены жирными линиями.

Важные характеристики предфрактального графа $G_{L}=\left(V_{L}, E_{L}\right)$ - количество его вершин $N=$ $N(n, L)=\left|V_{L}\right|$ и ребер $M=M(n, q, L)=\left|E_{L}\right|$ соответственно:

$N=N(n, L)=n^{L}$

$M(n, q, L)=q\left(1+n+n^{2}+\ldots+n^{L-1}\right)=q \frac{n^{L}-1}{n-1}$. 


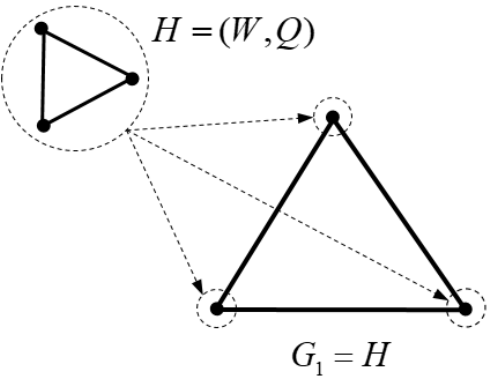

a)

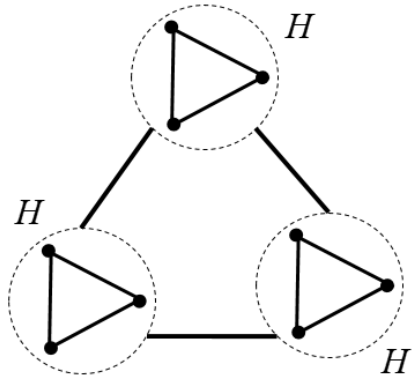

б)

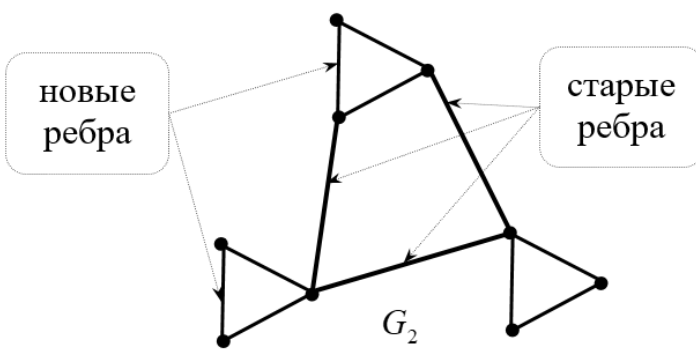

B)

Fig. 1. The operation of replacing the vertices of the graph $G_{1}$ with a seed $H$
Рис. 1. Операция замещения вершин графа $G_{1}$ затравкой $H$

В процессе порождения используются различные условия смежности, основные из них: смежность старых ребер произвольная (рис. 1.в); смежность старых ребер сохраняется (рис. 2); старые ребра не пересекаются (рис. 3).

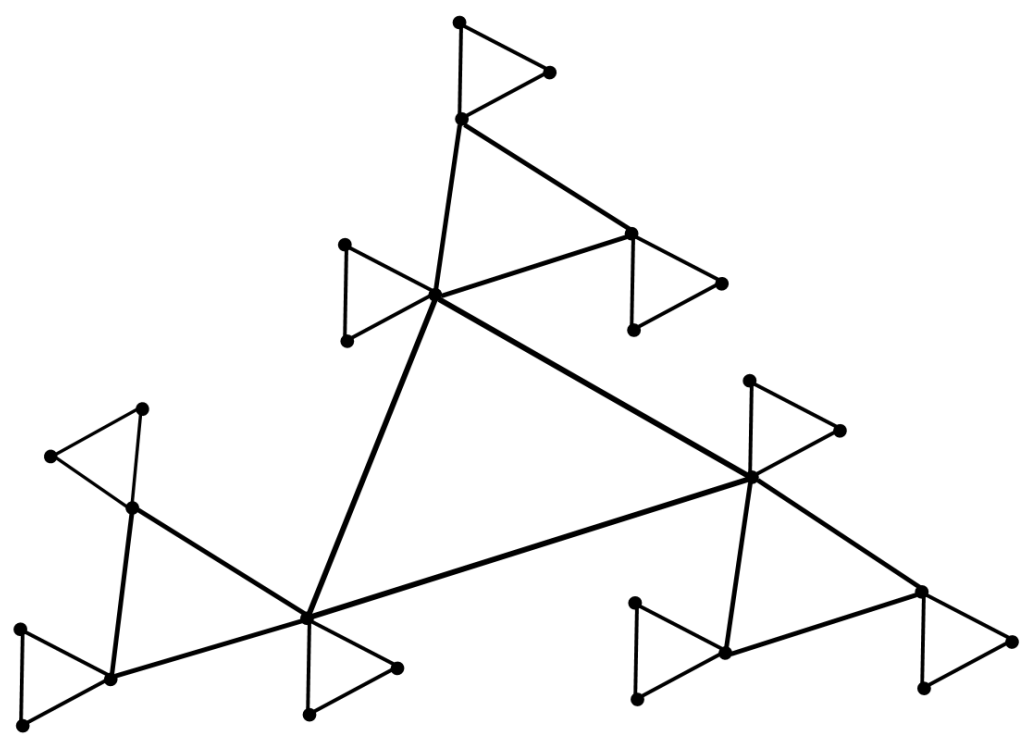

Fig. 2. Contiguity old edges preserved

Рис. 2. Смежность старых ребер сохраняется 
Условие сохранения смежности старых ребер применяется для старых ребер всех рангов. В некоторых случаях оговаривается сохранение смежности старых ребер одного или нескольких рангов, например, сохранение смежности старых ребер с чередованием.

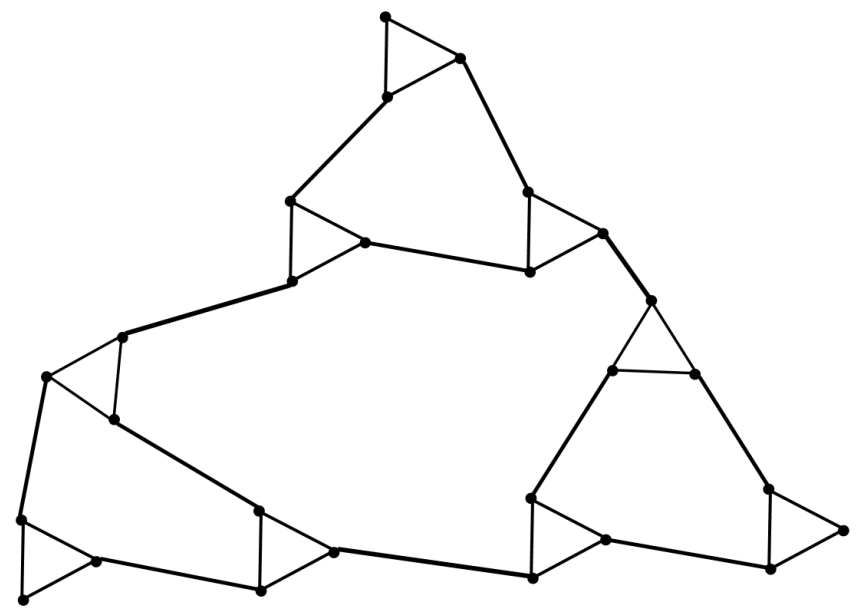

Fig. 3. Old edges are not crossed

Рис. 3. Старые ребра не пересекаются

Условие непересечения старых ребер, или, по-другому, отсутствие смежности применяется к старым ребрам всех рангов, в том числе старым ребрам разных рангов.

Ниже приводятся формулировки нескольких лемм, касающихся структурных особенностей предфрактальных графов.

ЛЕММА 1. Всякий предфрактальный граф $G_{l}, l \in[1,2, \ldots L]$ можно построить с непересекающимися старыми ребрами.

ЛЕММА 2. Предфрактальный граф $G_{l}, l \in[1,2, \ldots L]$, порожденный связной затравкой, является также связным.

Далее приводятся формулировки классических труднорешаемых задач, представленных в монографии [9]. После каждой задачи предлагается новая теорема для предфрактальных графов.

\section{1. Гамильтонов цикл}

ЗАДАЧА 1. Гамильтонов цикл.

УСЛОВИЕ. Задан граф $G=(V, E)$.

ВОПРОС. Имеется ли в $G$ гамильтонов цикл?

TEOРЕМА 1. Если на затравке $H$ имеется гамильтонов цикл, и между любой ее парой вершин имеется гамильтонова цепь, а старые ребра на предфрактальном графе не пересекаются, то на $G_{l}, l \in[1,2, \ldots L]$, существует гамильтонов цикл.

ДОКАЗАТЕЛЬСТВО.

На графе $G_{1}$ существует гамильтонов цикл $C_{1}=C_{1}^{1}$ по условию теоремы, так $G_{1}$ представляет собой затравку $H$. На следующем шаге ко всем вершинам $G_{1}$ применяется процедура 3 ВЗ и формируется $G_{2}$ при этом старые ребра первого ранга не пересекаются. Или по-другому к вершинам гамильтонова цикла $C_{1}$ применяется процедура 3 В3 и старые ребра первого ранга не пересекаются. Ребра первого ранга смежные в $C_{1}$ оказались разделенными затравками $H$. На каждой затравке выделяется гамильтонова цепь $C_{s}^{2}$ между ребрами первого ранга, которые были смежными в $C_{1}$. Таким образом, охвачены все вершины $G_{2}$ и построен гамильтонов цикл $C_{2}$.

Заместив вершины $C_{l}, l=2,3, \ldots, L-1$ затравками $H$ и выделив на них гамильтоновы цепи $C_{s}^{l+1}$, $s=1,2, \ldots, n^{l-1}$, сформированы гамильтоновы циклы $C_{l+1}$ на предфрактальных графах $G_{l+1}$. Таким образом, для каждого предфрактального графа $G_{l}$ ранга $l \in[1,2, \ldots, L]$ выделен гамильтонов цикл $C_{l}$. 
Условие теоремы о непересечении старых ребер позволяет на каждом шаге разделять гамильтонов цикл подграф-затравками и достраивать недостающие части гамильтоновыми цепями. Обратное условие сохранения смежности старых ребер не позволяет построить гамильтонов цикл далее графа $G_{1}$, так как затравки $H$ на каждом шаге порождения склеиваются к существующим вершинам, и цикл проходит общие вершины не менее двух раз. ТЕОРЕМА ДОКАЗАНА.

СЛЕДСТВИЕ 1.1. Если смежность старых ребер предфрактального графа $G_{l}, l \in[1,2, \ldots L]$ сохраняется, то на нем не существует гамильтонова цикла.

АЛГОРИТМ $\alpha_{1}$ ВЫДЕЛЕНИЯ ГАМИЛЬТОНОВА ЦИКЛА

ВХОД: взвешенный предфрактальный граф $G_{L}$.

ВЫХОД: гамильтонов цикл $C=\left(V, E_{c}\right)$.

ШАГ 1. Применяется процедура поиска гамильтонова цикла к $G_{1}$. На выходе процедуры получен гамильтонов цикл $C_{1}=C_{1}^{1}$.

ДЛЯ ВСЕХ $l=2,3, \ldots, L$ ВЫПОЛНИТЬ:

ШАГ $l$. ДЛЯ ВСЕХ $s=1,2, \ldots, n^{l-1}$ ВЫПОЛНИТЬ:

ШАГ l.s. Для каждой $s$-ой затравки $l$-го ранга $H$ выполнить процедуру поиска гамильтоновой цепи $C_{s}^{l}$ между ребрами $(l-1)$-го ранга гамильтонового цикла $C_{l-1}$.

На выходе шага $l=2,3, \ldots, L$ выделен гамильтонов цикл $C_{l}$.

ПРОЦЕДУРА ГАМИЛЬТОНОВ ЦИКЛ

ВХОД: взвешенный граф $G=(V, E)$.

ВЫХОД: гамильтонов цикл $C_{s}=\left(V, E_{s}\right)$.

ПРОЦЕДУРА ГАМИЛЬТОНОВА ЦЕПЬ

ВХОД: взвешенный граф $G=(V, E)$.

ВЫХОД: гамильтонова цепь $C_{s}=\left(V, E_{s}\right)$.

СЛЕДСТВИЕ 1.2. При выполнении условий теоремы о существовании параметризованный алгоритм выделяет гамильтонов цикл на предфрактальном графе $G_{l}$ за время $O(c \cdot N)$, где $N=n^{l}$ и $c=(n-1) !$

Используя переборный алгоритм, выделение гамильтонова цикла или цепи на затравке $H$ в худшем случае потребует выполнения $(n-1)$ ! операций. Всего на предфрактальном графе $G_{l}$ присутствует $\left(n^{l}-1\right) /(n-1) \leq n^{l}=N$ затравок.

ПРИМЕЧАНИЕ 1.1. В теореме 1 и следствии 1.1 рассматриваются два противоположных случая - старые ребра не пересекаются и смежность старых ребер сохраняется. При этом условие “старые ребра не пересекаются” означает, что старые ребра любых рангов не являются смежными между собой. Исследование остальных случаев является предметом дальнейших научных изысканий, в том числе в виде перечня теорем, которые покрывали бы всевозможные случаи порождения предфрактального графа.

ПРИМЕЧАНИЕ 1.2. В следствии 1.1 указанный алгоритм полиномиален относительно $N$ при фиксировании параметра $n$. Это примечание также верно для следствий 2.1 и 3.1 .

ПРИМЕЧАНИЕ 1.3. Описание алгоритма $\alpha_{1}$ приведено в качестве примера. Алгоритмы разрабатываются в тесной связи с доказательствами соответствующих теорем. Далее для некоторых теорем указана трудоемкость алгоритмов без их описания.

\section{2. Остов с максимальным числом висячих вершин}

ЗАДАЧА 2. Остов с максимальным числом висячих вершин.

УСЛОВИЕ. Заданы граф $G=(V, E)$ и положительное целое число $K \leqslant|V|$.

ВОПРОС. Существует ли в графе $G$ остов, у которого не менее $K$ вершин степени 1 ? 
TEОРЕМА 2. Если на затравке $H$ существует остов, у которого не менее $k \leq n$ висячих вершин и смежность старых ребер сохраняется, то на предфрактальном графе $G_{l}, l \in[1,2, \ldots L]$ существует остов, у которого не менее $K$ висячих вершин, где $K=(k-1) n^{l-1}$.

\section{ДОКАЗАТЕЛЬСТВО.}

На графе $G_{1}$ существует остов $T_{1}=T_{1}^{1}$, у которого не менее $k \leq n$ висячих вершин по условию теоремы, так $G_{1}$ представляет собой затравку $H$. На следующем шаге ко всем вершинам $G_{1}$ применяется процедура ЗВЗ и формируется $G_{2}$ с сохранением смежности старых ребер. Или по-другому к вершинам остова $T_{1}$ применяется процедура ЗВ3 с сохранением смежности старых ребер. Поскольку все вершины $T_{1}$ замещаются затравками, то ни одна такая вершина не является висячей в $G_{2}$. Выделяя остовы $T_{s}^{2}$ на затравках второго ранга и добавляя остов $T_{1}$, формируется остов $T_{2}$ на $G_{2}$. В остове $T_{2}$ висячими могут являться только висячие вершины в $T_{s}^{2}$. На любой затравке не менее $k \leq n$ висячих вершин. Остовы $T_{s}^{2}$ фактически склеиваются с вершинами $T_{1}$ одной из своих вершин. В худшем случае остов $T_{s}^{2}$ может склеиваться своей висячей вершиной, тогда количество висячих вершин остова $T_{2}$ в $G_{2}$ равно произведению числа висячих вершин без одной на затравке на число затравок второго ранга: $(k-1) n$.

Процедура порождения графов траектории $G_{l}, l=2,3, \ldots, L$ затравками $H$ и выделение на них остовов $T_{s}^{l}, s=1,2, \ldots, n^{l-1}$ формирует остовы $T_{l}$ на предфрактальных графах $G_{l}$. Таким образом, для каждого предфрактального графа $G_{l}$ ранга $l \in[1,2, \ldots L]$ выделен остов $T_{l}$ с не менее $K=(k-1) n^{l-1}$ вершин. ТЕОРЕМА ДОКАЗАНА.

СЛЕДСТВИЕ 2.1. При выполнении условий теоремы о существовании параметризованный алгоритм выделяет на предфрактальном графе $G_{l}$ остов, у которого не менее $K=(k-1) n^{l-1}$ висячих вершин, за время $O(c \cdot N)$, где $N=n^{l}$ и $c=2^{n}$.

Выделение остовных деревьев с максимальным числом висячих вершин на затравках может осуществляться любым известным алгоритмом, в худшем случае потребует выполнения $2^{n}$ операций. Всего на предфрактальном графе $G_{l}$ присутствует $\left(n^{l}-1\right) /(n-1) \leq n^{l}=N$ затравок.

\section{3. Монохроматический треугольник}

ЗАДАЧА 3. Монохроматический треугольник.

УСЛОВИЕ. Задан граф $G=(V, E)$.

ВОПРОС. Существует ли разбиение множества $E$ на два непересекающихся множества $E_{1}$ и $E_{2}$, такие, что ни один из графов $G_{1}=\left(V, E_{1}\right)$ или $G_{2}=\left(V, E_{2}\right)$ не содержит треугольника?

TEOPEMA 3.1. На предфрактальном графе $G_{l}, l \in[1,2, \ldots L]$ не существует треугольников, состоящих из ребер соседних рангов.

ДОКАЗАТЕЛЬСТВО.

Рассматривается предфрактальный граф второго ранга $G_{2}$. Предположим, что существует треугольник, состоящий из двух ребер первого ранга и одного ребра второго ранга. Применим операцию стягивания затравок, обратную ЗВ3, тогда два смежных ребра первого ранга стягиваются в кратное ребро, что противоречит определению предфрактального графа. Предположим, что существует треугольник, состоящий из двух ребер второго ранга и одного ребра первого ранга. Применив операцию стягивания затравок, два смежных ребра второго ранга стягиваются в вершину, а ребро первого ранга представляет собой петлю, что также противоречит определению предфрактального графа.

Рассматривается предфрактальный граф $l$-го ранга $G_{l}$. Предположим, что существует треугольник, состоящий из двух ребер $(l-1)$-го ранга и одного ребра $l$-го ранга. Применив операцию стягивания затравок $l$-го ранга, получим, что два смежных ребра $(l-1)$-го ранга стягиваются в кратное ребро, что противоречит определению предфрактального графа. Предположим, что существует треугольник, состоящий из двух ребер $l$-го ранга и одного ребра $(l-1)$-го ранга. Применяя операцию стягивания затравок $l$-го ранга, два смежных ребра $l$-го ранга стягиваются в вершину, а ребро 
$(l-1)$-го ранга представляет собой петлю, что также противоречит определению предфрактального графа. Таким образом, на предфрактальном графе $G_{l}$ невозможно построить треугольник из ребер соседних рангов. ТЕОРЕМА ДОКАЗАНА.

TEOPEMA 3.2. На предфрактальном графе $G_{l}, l \in[1,2, \ldots L]$ не существует треугольников, состоящих из ребер разных рангов.

ДОКАЗАТЕЛЬСТВО.

На предфрактальном графе $G_{l}$ невозможно построить треугольник из ребер соседних рангов, как было доказано в предыдущей теореме.

Рассматривается предфрактальный граф $l$-го ранга $G_{l}$. Предположим, что существует треугольник, состоящий из двух ребер $l_{1}$-го ранга и одного ребра $l_{2}$-го ранга, $l_{1}<l_{2}$ и $l_{1}, l_{2} \in[1,2, \ldots, L]$. Применив операцию стягивания затравок $l_{2}$-го ранга, получим, что два смежных ребра $l_{1}$-го ранга стягиваются в кратное ребро, что противоречит определению предфрактального графа. Предположим, что существует треугольник, состоящий из двух ребер $l_{2}$-го ранга и одного ребра $l_{1}$-го ранга, $l_{1}<l_{2}$. Применяя операцию стягивания затравок $l_{2}$-го ранга, два смежных ребра $l_{2}$-го ранга стягиваются в вершину, а ребро $l_{1}$-го ранга представляет собой петлю, что также противоречит определению предфрактального графа. Теперь предположим, существует треугольник, состоящий из одного ребpa $l_{1}$-го ранга, одного ребра $l_{2}$-го ранга и одного ребра $l_{3}$-го ранга $l_{1}<l_{2}<l_{3}$ и $l_{1}, l_{2}, l_{3} \in[1,2, \ldots, L]$. Применяя операцию стягивания затравок $l_{3}$-го ранга получим, что два смежных ребра $l_{1}$-го и $l_{2}$-го рангов стягиваются в кратное ребро, что противоречит процедуре порождения предфрактального графа и выводит из класса предфрактальных графов замкнутых относительно операции ЗВЗ. Таким образом, на предфрактальном графе $G_{l}$ невозможно построить треугольник из ребер разных рангов. ТЕОРЕМА ДОКАЗАНА.

ТЕОРЕМА 3.3. Если затравка $H$ - монохроматический треугольник, то предфрактальный граф $G_{l}, l \in[1,2, \ldots L]$ также монохроматический треугольник.

ДОКАЗАТЕЛЬСТВО.

Предфрактальный граф $G_{1}$ является монохроматическим треугольником по условию теоремы, так как представляет собой затравку $H$. То есть $E_{1}$ можно разбить на два непересекающихся подмножества $E_{1}^{1}$ и $E_{2}^{1}$, каждое из которых не содержит треугольников.

На графе второго ранга $G_{2}$ к графу $G_{1}$ добавляются затравки $H$, множество ребер каждой из них можно разбить на два непересекающихся подмножества $E_{s, 1}^{2}$ и $E_{s, 2}^{2}$. Объединяем все $E_{s, 1}^{2}$ в одно множество $E^{\prime}$, а все $E_{s, 2}^{2}$ во второе множество $E^{\prime \prime}$. Новые треугольники после объединения не могут появиться, так как ребра затравок второго ранга не пересекаются. Добавляя $E_{1}^{1}$ к $E^{\prime}$, а $E_{2}^{1}$ к $E^{\prime \prime}$ также новых треугольников не образуется, в силу выполнения теоремы о невозможности существования треугольников из ребер разных рангов. Таким образом, ребра $G_{2}$ разделены на два непересекающихся подмножества $E^{\prime}$ и $E^{\prime \prime}$, не содержащих треугольников.

Рассматривается предфрактальный граф $l$-го ранга $G_{l}, l \in[2,3, \ldots L]$, полученный из $G_{l-1}$ операцией 3В3 H. Ребра каждой затравки $l$-го ранга можно разбить на два непересекающихся подмножества $E_{s, 1}^{l}$ и $E_{s, 2}^{l}$. Объединяем все $E_{s, 1}^{l}$ в одно множество $E^{\prime}$, а все $E_{s, 2}^{l}$ во второе множество $E^{\prime \prime}$. Новых треугольников после объединения не может появиться, так как ребра затравок $l$-го ранга не пересекаются. Добавляя $E_{s, 1}^{l-1} \kappa E^{\prime}$, а $E_{s, 2}^{l-1}$ к $E^{\prime \prime}$ также новых треугольников не образуется, в силу выполнения теоремы о невозможности существования треугольников из ребер разных рангов. Получаем, что ребра $G_{l}$ разделены на два непересекающихся подмножества $E^{\prime}$ и $E^{\prime \prime}$, не содержащих треугольников.

Таким образом, предложена процедура разделения множества ребер предфрактального графа $G_{l}, l \in[1,2, \ldots L]$ на два непересекающихся подмножества $E^{\prime}$ и $E^{\prime \prime}$, не содержащих треугольников. Процедура проводится пошагово от графа $G_{1}$ к $G_{2}$ и так далее до $G_{l}$ включительно. TEOPEMA ДОКАЗАНА. 
СЛЕДСТВИЕ 3.1. При выполнении условий теоремы о монохроматическом треугольнике параметризованный алгоритм разделяет множество ребер предфрактального графа $G_{l}, l \in[1,2, \ldots L]$ на два непересекающихся подмножества, не содержащих треугольники, за время $O(c \cdot N)$, где $N=n^{l}$ и $c=2^{n}$.

Используя переборный алгоритм, разделение множества ребер на затравке $H$ в худшем случае потребует выполнения $2^{n}$ операций. Всего на предфрактальном графе $G_{l}$ присутствует $\left(n^{l}-1\right) /(n-1) \leq$ $n^{l}=N$ затравок.

\section{4. Клика}

ЗАДАЧА 4. Клика.

УСЛОВИЕ. Заданы граф $G=(V, E)$ и положительное целое число $K \leqslant|V|$.

ВОПРОС. Верно ли, что $G$ содержит клику размера не менее $K$ ? Иными словами, существует ли подмножество $V^{\prime} \subseteq V$ такое, что $\left|V^{\prime}\right| \geqslant K$ и любые две вершины в $V^{\prime}$ соединены ребром из $E$ ?

TEOPEMA 4. Если на затравке $H$ существует клика размером не менее $k \leq n$, то на предфрактальном графе $G_{l}, l \in[1,2, \ldots L]$ существует клика размером не менее $k$.

ДОКАЗАТЕЛЬСТВО.

На предфрактальном графе $G_{l}$ на затравках $l$-го ранга существуют клики размером не менее $k$ в силу условия теоремы.

Клики размера $k$, выделенные на затравках $l$-го ранга, нельзя увеличить за счет старых ребер предыдущих рангов. Клика состоит из треугольников и для того, чтобы перейти к клике размера $(k+1)$, необходимо использовать ребра предыдущих рангов (ребра разных затравок одинакового ранга не пересекаются). Но построить треугольники из ребер разных рангов нельзя в силу ограничений теоремы о существовании треугольников, состоящих из ребер разных рангов. Таким образом, выбирая любую клику размера $k$ на затравках, невозможно увеличить ее до размера $(k+1)$ на предфрактальном графе. ТЕОРЕМА ДОКАЗАНА.

\section{5. Независимое множество}

ЗАДАЧА 5. Независимое множество.

УСЛОВИЕ. Заданы граф $G=(V, E)$ и положительное целое число $K \leqslant|V|$.

ВОПРОС. Верно ли, что $G$ содержит независимое множество мощности не менее $К$ ? Иными словами, верно ли, что существует подмножество $V^{\prime} \subseteq V$ такое, что $\left|V^{\prime}\right| \geqslant K$ и никакие две вершины в $V^{\prime}$ не соединены ребром из $E$ ?

TEОРЕМА 5. Если затравка $H$ содержит независимое множество мощности не менее $k \leq n$ и смежность старых ребер сохраняется, то предфрактальный граф $G_{l}, l \in[1,2, \ldots L]$ содержит независимое множество мощности $K \leq k n^{l-1}$.

ДОКАЗАТЕЛЬСТВО.

По условию теоремы $G_{1}$ содержит независимое множество $V_{1}^{\prime}$ мощности не менее $k$, так как представляет собой затравку $H$. Выделяя независимое множество $V_{1}^{\prime}$ на $G_{1}$, смежные вершины перемещаются во множество $V_{1}^{\prime \prime}$. На следующем шаге из $G_{1}$ удаляются вершины $V_{1}^{\prime \prime}$ и перемещаются в $V_{2}^{\prime \prime}$, тогда в $G_{2}^{\prime}$ все новые затравки второго ранга не пересекаются (в силу сохранения смежности старых ребер), так как вершины со смежными старыми ребрами первого ранга удалены. На урезанных затравках $G_{2}^{\prime}$ выделяются независимые множества и сохраняются в $V_{2}^{\prime}$, а все смежные вершины помещаются в $V_{2}^{\prime \prime}$. Множество $V_{2}^{\prime \prime}$ содержит в себе удаленные вершины на первом шаге $G_{1}$ и втором шаге $G_{2}$.

На следующем шаге из $G_{3}$ удаляются вершины $V_{2}^{\prime \prime}$ и перемещаются в $V_{3}^{\prime \prime}$, тогда в $G_{3}^{\prime}$ все новые затравки третьего ранга не пересекаются, так как вершины со смежными старыми ребрами второго ранга удалены. На урезанных затравках $G_{3}^{\prime}$ выделяются независимые множества и сохраняются в $V_{3}^{\prime}$, 
а все смежные вершины помещаются в $V_{3}^{\prime \prime}$. Множество $V_{3}^{\prime \prime}$ содержит в себе удаленные вершины на первом шаге $G_{1}$, втором шаге $G_{2}$ и третьем шаге $G_{3}$.

Процедура порождения графов траектории $G_{l}, l=2,3, \ldots L$ затравками $H$ формирует на предфрактальных графах $G_{l}$ независимые множества $V_{l}^{\prime}$. Таким образом, для каждого предфрактального графа $G_{l}$ ранга $l \in[1,2, \ldots L]$ выделено независимое множество $V_{l}^{\prime}$ мощности $K \leq k n^{l-1}$. TEOPEMA ДОКАЗАНА.

\section{Заключение}

В работе рассмотрены и исследованы NP-полные задачи на одном из классов динамических графов - предфрактальных графах. По каждой из задач приведены условия разрешимости, при которых для некоторых подзадач возможно получить ответ о существовании и построить полиномиальные (при фиксировании числа вершин затравки) алгоритмы поиска решений. Ряд следствий обобщают полученные результаты, либо приводят условия - отсутствия решения. Например, для задачи выделения Гамильтонова цикла, приведено условие отсутствия решения на предфрактальном графе. Для некоторых задач приведены расчеты вычислительных сложностей поиска решений.

В данной работе приведено исследование малой части из числа известных NP-полных задач. Исследование всего набора задач и выделение условий разрешимости для класса динамических графов - предфрактальных графов, позволит по-сути говорить о выделении целого подкласса графов с условиями разрешимости NP-полных задач.

Разработка полиномиальных (при фиксировании числа вершин затравки) алгоритмов для динамических графов позволит решать с улучшенными характеристиками такие известные прикладные задачи как выделение подграфов в больших динамических сетях - выделение сообществ в социальных сетях; решение многокритериальных задач в транспортно-логистических системах большой размерности; поиск и выделение ddos-атак в криптовалютных системах и многие другие задачи.

\section{References}

[1] A. Kochkarov, R. Kochkarov, and G. Malinetskii, "Issues of dynamic graph theory", Computational Mathematics and Mathematical Physics, vol. 55, no. 9, pp. 1590-1596, 2015.

[2] S. Pupyrev and A. Tikhonov, "The Analysis of Complex Networks with Dynamic Graph Visualization", Modeling and Analysis of Information Systems, vol. 17, no. 1, pp. 117-135, 2010.

[3] Y. Belov and S. Vovchok, "Generation of a Social Network Graph by Using Apache Spark", Modeling and Analysis of Information Systems, vol. 23, no. 6, pp. 777-783, 2016.

[4] S. Morzhov and V. Sokolov, "An Effective Algorithm for Collision Resolution in Security Policy Rules", Modeling and Analysis of Information Systems, vol. 26, no. 1, pp. 75-89, 2019.

[5] A. M. Kochkarov, V. A. Perepelitsa, and I. V. Sergienko, Recognition of fractal graphs. Algorithmic approach. RAS SAO, 1998.

[6] R. A. Kochkarov, "Problems of multicriteria optimization on multi-weighted prefractal graphs", no. 17, pp. 319-328, 2014.

[7] F. Harary, Graph theory. Addison-Wesley Pub. Co., 1969.

[8] M. A. Iordanskii, "Constructive Classification of Graphs", Modeling and Analysis of Information Systems, vol. 19, no. 4, pp. 144-153, 2012.

[9] M. R. Garey and D. S. Johnson, Computers and Intractability: A Guide to the Theory of NP-Completeness. W. H. Freeman and Company, 1979. 